\title{
Macular Structure Recovery after Surgery for Optic Disc Pit Maculopathy
}

\author{
Yuri Nishiyama Yuji Yoshikawa Masayuki Shibuya Junji Kanno \\ Kimitake Ozaki Yu Sakaki Takeshi Katsumoto Jun Makita \\ Takuhei Shoji Kei Shinoda \\ Department of Ophthalmology, Faculty of Medicine, Saitama Medical University, \\ Saitama, Japan
}

\section{Keywords}

Optic disc pit · Optic disc pit maculopathy · Outer layer hole $\cdot$ Intraretinal fluid · Serous retinal detachment

\begin{abstract}
The present report aimed to describe the macular structure's recovery process in a case of optic disc pit maculopathy (ODP-M) with outer layer hole following pars plana vitrectomy (PPV) with juxtapapillary laser treatment (JPL). We performed repeated optical coherence tomography (OCT) examinations to evaluate the macular structural changes. An 80-year-old man presented with distorted vision and decreased visual acuity (VA) in his left eye, experienced for 1 year, prior to presentation. Fundus examination and OCT showed intraretinal fluid (IRF) in the inner and outer retinal layers. Serous retinal detachment (SRD) with an outer layer hole in the macula was also evident. The IRF was connected to the optic disc; however, the SRD was isolated. Best-corrected VA was 20/100. PPV combined with JPL was performed. Posterior vitreous detachment creation and tamponade with sulfur hexafluoride was performed. Postoperatively, the inner retinal IRF at the fovea disappeared. The outer layer hole gradually closed and had completely disappeared 1 month postoperatively. After resolution of the outer layer hole, SRD reduced gradually and disappeared 8 months postoperatively, although the macular outer retinal IRF remained. The outer retinal IRF had partially resolved by the 18th postoperative month. Macular structure was completely recovered 31 months postoperatively, with an improved VA
\end{abstract}

KARGER
Kei Shinoda

Department of Ophthalmology

Faculty of Medicine, Saitama Medical University

38 Moro-Hongo Moroyama-machi, Iruma-gun, Saitama 350-0495 (Japan)

E-Mail shinok@saitama-med.ac.jp 
of 20/20. In conclusion, SRD might be associated with outer retinal IRF and outer layer holes. In cases of ODP-M, outer layer holes might induce optic disc-isolated SRD.

(C) 2019 The Author(s)

Published by S. Karger AG, Basel

\section{Introduction}

Optic disc pits (ODPs) were first described in 1882 by Wiethe [1] as a congenital cavity of the optic nerve; it occurs in less than 1 per 10,000 individuals and is bilateral in up to $15 \%$ of cases [2, 3]. Maculopathy associated with ODP (ODP-M) is reported in $25-93 \%$ of adult ODP patients [4-7]. However, some ODP-M cases occur without pits [8] or occult pits in patients with glaucoma [9]. The development process of ODP-M and the source of its fluid are unknown.

Lincoff et al. [10] reported that 14 of 15 eyes with ODP-M had outer layer holes and hypothesized that subretinal fluid (SRF) in the macular region entered through an inner schisislike cavity through an outer layer hole. This theory has been supported to varying degrees by optical coherence tomography (OCT) findings $[4,11,12]$. However, other reports showed detail cases of macular detachment without evidence of an inner retinal schisis cavity [13] or outer layer holes [14]. As such, the significance of the outer layer hole remains controversial, and the mechanism of SRF accumulation remains unknown. This study aims to shed light on these 2 unanswered questions.

For this purpose, we used OCT to observe long-term recovery after surgery for intraretinal fluid (IRF) accumulation, outer layer hole, and serous retinal detachment (SRD) in an eye with ODP-M.

\section{Case Report}

An 80-year-old man complained of distorted vision and decreased visual acuity (VA) in his left eye that had lasted for 1 year. Fundus examination and OCT showed IRF in the inner and outer retinal layers and SRD with an outer layer hole in the macula. The IRF was connected to the optic disc, but the SRD was isolated from the optic disc (Fig. 1). Best-corrected VA was 20/100 Snellen acuity. Pars plana vitrectomy combined with juxtapapillary laser treatment was performed. Posterior vitreous detachment creation and tamponade with sulfur hexafluoride was performed. Postoperatively, the inner retinal IRF at the fovea was resolved. The outer layer hole closed gradually and had completely disappeared by 1 postoperative month (Fig. 1, Fig. 2). After the outer layer hole had resolved, SRD gradually reduced and had completely disappeared 8 months postoperatively, although the macular area outer retinal IRF remained (Fig. 2). The outer retinal IRF resolved at postoperative 18 months. Macular structure was completely recovered 31 months postoperatively, with improved VA of 20/20 (Fig. 3).

\section{Discussion}

In this report, we have described the recovery process of the macular structure with OCT and suggested that the outer layer hole might contribute to the developmental process of SRD isolated from the optic disc in a case of ODP-M. 
Imamura et al. [14] reported that the fluid from an optic nerve pit could directly enter different retinal layers. In their study, 6 of 11 eyes with SRF (55\%) had SRF in communication with the optic nerve pit. However, there were cases where the SRF was isolated from the optic disc and other cases with residual localized SRD and an outer layer hole.

Lincoff et al. [10] showed that 14 of 15 eyes had an outer layer hole and hypothesized that SRF developed from the outer layer hole. Contrastingly, Imamura et al. [14] reported only 3 of 11 eyes with outer layer holes and argued that they were not necessary for retinal detachment. They posited that IRF could percolate the subretinal space without an outer layer hole.

In the present case, SRD was not in direct contact with the optic disc and coexisted with an outer layer hole. The outer layer hole closed gradually after surgery, but IRF and SRD persisted. The SRD completely disappeared after the outer layer hole closure, although the IRF remained.

Previous case reports $[14,15]$ have documented cases of ODP-M without SRD. Here, we propose that SRF originates as IRF, entering through an outer layer hole or due to IRF-induced mechanical stress that forces fluid into the subretinal space. Although the exact trigger of SRD is unknown, fixing the outer layer hole in the present case helped to resolve the isolated SRD. Consequently, we present a hypothesis that the outer layer hole contributed to the SRD development.

In conclusion, the SRD might be associated with the outer retinal IRF via outer layer holes. An outer layer hole might contribute to the development of optic disc-isolated SRD in cases of ODP-M. Further research and case studies are necessary to confirm and expand on our findings.

\section{Acknowledgements}

The authors thank Editage (https://www.editage.jp) for English language editing.

\section{Statement of Ethics}

Written consent to publish this case has not been obtained. This report does not contain any personal identifying information.

\section{Disclosure Statement}

The authors have no conflicts of interest to declare.

\section{Funding Sources}

This study was supported in part by the Japan Society for the Promotion of Science (JSPS; KAKENHI grant number: 17K11430). 


\section{Author Contributions}

M.S. treated the patient, including surgery and follow-up. Y.Y., T.S., Y.N., K.O., Y.S., T.K., J.M., K.S., and M.S. collected the data, analyzed the ophthalmological findings, and gave critical suggestions. Y.N., Y.Y., J.K., and K.S. prepared the figures. Y.Y. prepared the report and K.S., T.S., and M.S. revised and finalized it. All authors agree to be accountable for all aspects of the work. Patient anonymity is preserved. All authors attest that they meet the current ICMJE criteria for authorship.

\section{References}

1 Wiethe T. Ein Fall von angeborener Deformität der Sehnervenpapille. Arch Augenheilkd. 1882;11:14-9.

2 Kranenburg EW. Crater-like holes in the optic disc and central serous retinopathy. Arch Ophthalmol. 1960 Dec;64(6):912-24.

3 Georgalas I, Ladas I, Georgopoulos G, Petrou P. Optic disc pit: a review. Graefes Arch Clin Exp Ophthalmol. 2011 Aug;249(8):1113-22.

4 Krivoy D, Gentile R, Liebmann JM, Stegman Z, Rosen R, Walsh JB, et al. Imaging congenital optic disc pits and associated maculopathy using optical coherence tomography. Arch Ophthalmol. 1996 Feb;114(2):165-70.

5 Sobol WM, Blodi CF, Folk JC, Weingeist TA. Long-term visual outcome in patients with optic nerve pit and serous retinal detachment of the macula. Ophthalmology. 1990 Nov;97(11):1539-42.

6 Theodossiadis G, Theodossiadis P, Malias J, Moschos M, Moschos M. Preoperative and postoperative assessment by multifocal electroretinography in the management of optic disc pits with serous macular detachment. Ophthalmology. 2002 Dec;109(12):2295-302.

7 Ghosh YK, Banerjee S, Konstantinidis A, Athanasiadis I, Kirkby GR, Tyagi AK. Surgical management of optic disc pit associated maculopathy. Eur J Ophthalmol. 2008 Jan-Feb;18(1):142-6.

8 Spaide RF, Costa DL, Huang SJ. Macular schisis in a patient without an optic disk pit optical coherence tomographic findings. Retina. 2003 Apr;23(2):238-40.

9 Nagesha CK, Ganne P. Occult Optic Disc Pit Maculopathy in a Glaucomatous Disc. Middle East Afr J Ophthalmol. 2017 Jul-Sep;24(3):165-6.

10 Lincoff H, Lopez R, Kreissig I, Yannuzzi L, Cox M, Burton T. Retinoschisis associated with optic nerve pits. Arch Ophthalmol. 1988 Jan;106(1):61-7.

11 Karacorlu SA, Karacorlu M, Ozdemir H, Burumcek E, Esgin H. Optical coherence tomography in optic pit maculopathy. Int Ophthalmol. 2007 Oct;27(5):293-7.

12 García-Arumí J, Guraya BC, Espax AB, Castillo VM, Ramsay LS, Motta RM. Optical coherence tomography in optic pit maculopathy managed with vitrectomy-laser-gas. Graefes Arch Clin Exp Ophthalmol. 2004 Oct;242(10):819-26.

13 Moon SJ, Kim JE, Spaide RF. Optic pit maculopathy without inner retinal schisis cavity. Retina. 2006 Jan;26(1):113-6.

14 Imamura Y, Zweifel SA, Fujiwara T, Freund KB, Spaide RF. High-resolution optical coherence tomography findings in optic pit maculopathy. Retina. 2010 Jul-Aug;30(7):1104-12.

15 Chen FK, Acheson JF, Franks WA, Da Cruz L. Microperimetry evidence of functional improvement after vitrectomy for optic disc pit-related intraretinal fluid without serous detachment. Clin Exp Ophthalmol. 2011 Sep-0ct;39(7):707-10. 


\section{Case Reports in Ophthalmology}
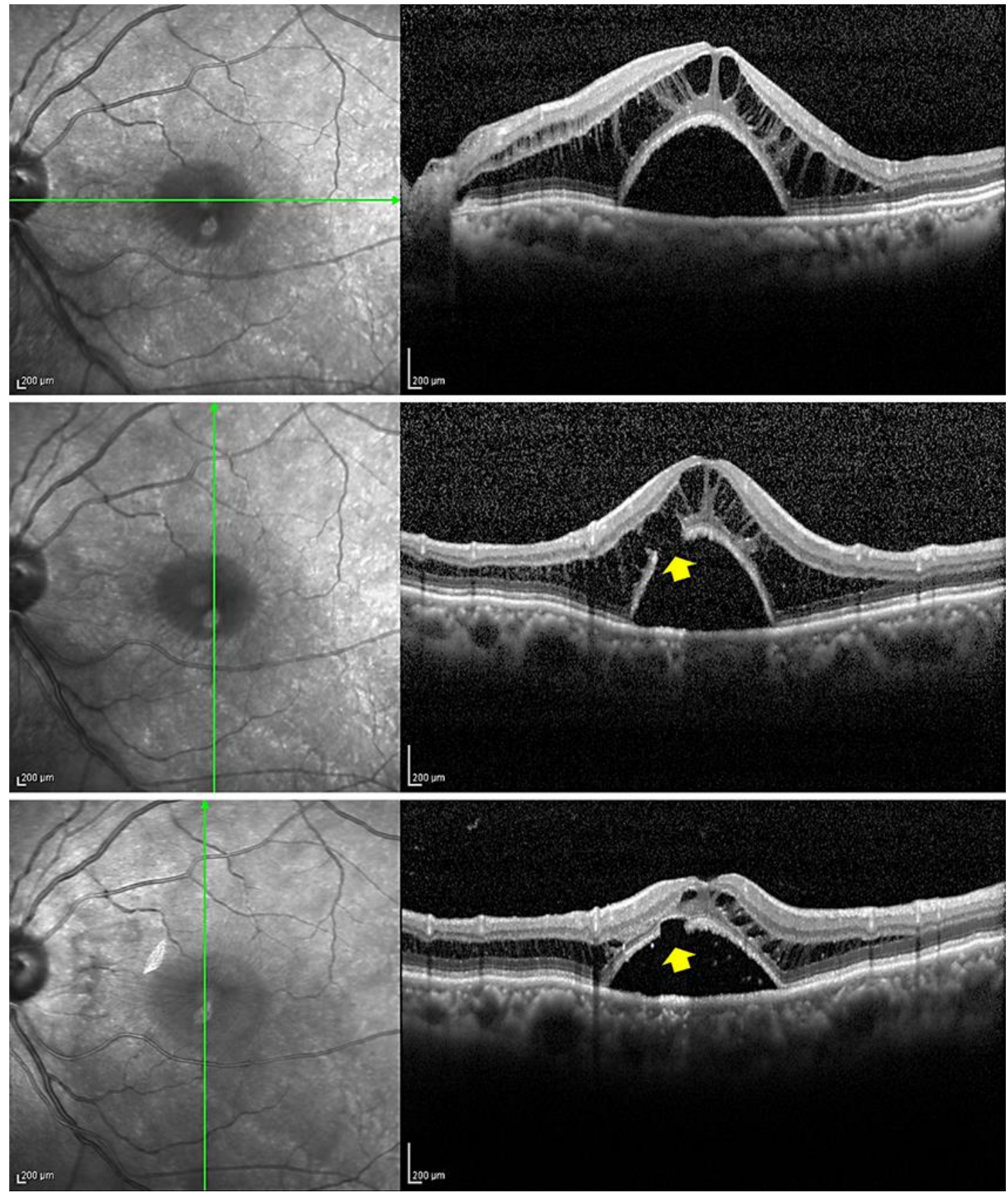

Fig. 1. Initial OCT and short-term follow-up OCT scans. Horizontal OCT scan showing IRF at the inner and outer nuclear layers and SRD. The IRF was connected to the optic disc, but the SRD was isolated from the optic disc. Vertical OCT scan shows the outer layer hole (yellow arrow; middle and lower panel), which connected outer retinal IRF to SRD. The outer layer hole closed as the foveal outer nuclear layer IRF reduced (lower panel, 1 week after surgery). Extrafoveal IRF and SRD persisted. OCT, optical coherence tomography; IRF, intraretinal fluid; SRD, serous retinal detachment. 


\section{Case Reports in Ophthalmology}
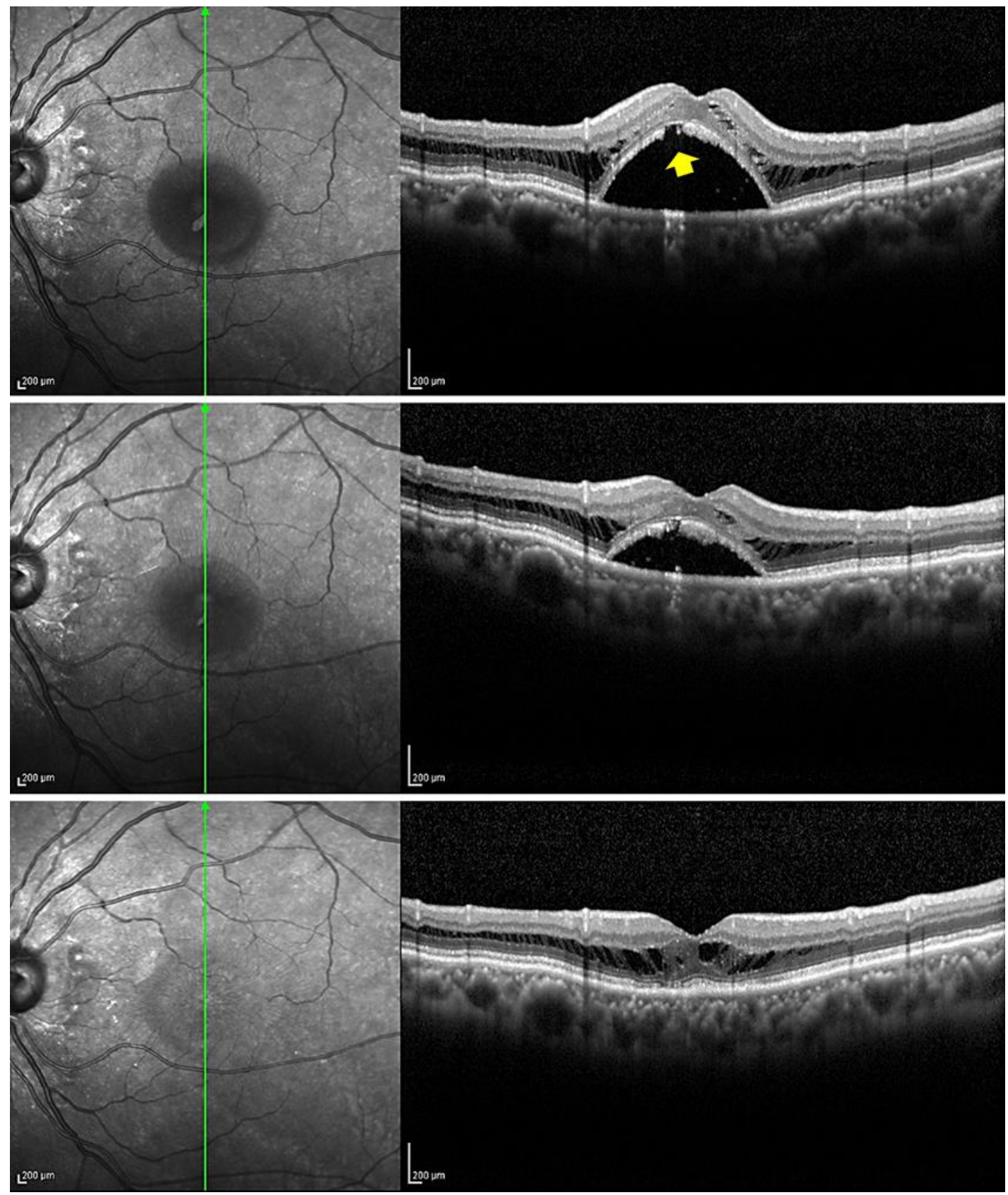

Fig. 2. Long-term follow-up OCT scans. One month after surgery, the outer layer hole was completely closed (upper panel). Then, 3 months after surgery, SRD had partially resolved (middle panel). Despite the persistence of outer nuclear layer IRF, SRD had completely disappeared by the 8th postoperative month (lower panel). OCT, optical coherence tomography; IRF, intraretinal fluid; SRD, serous retinal detachment. 


\section{Case Reports in Ophthalmology}
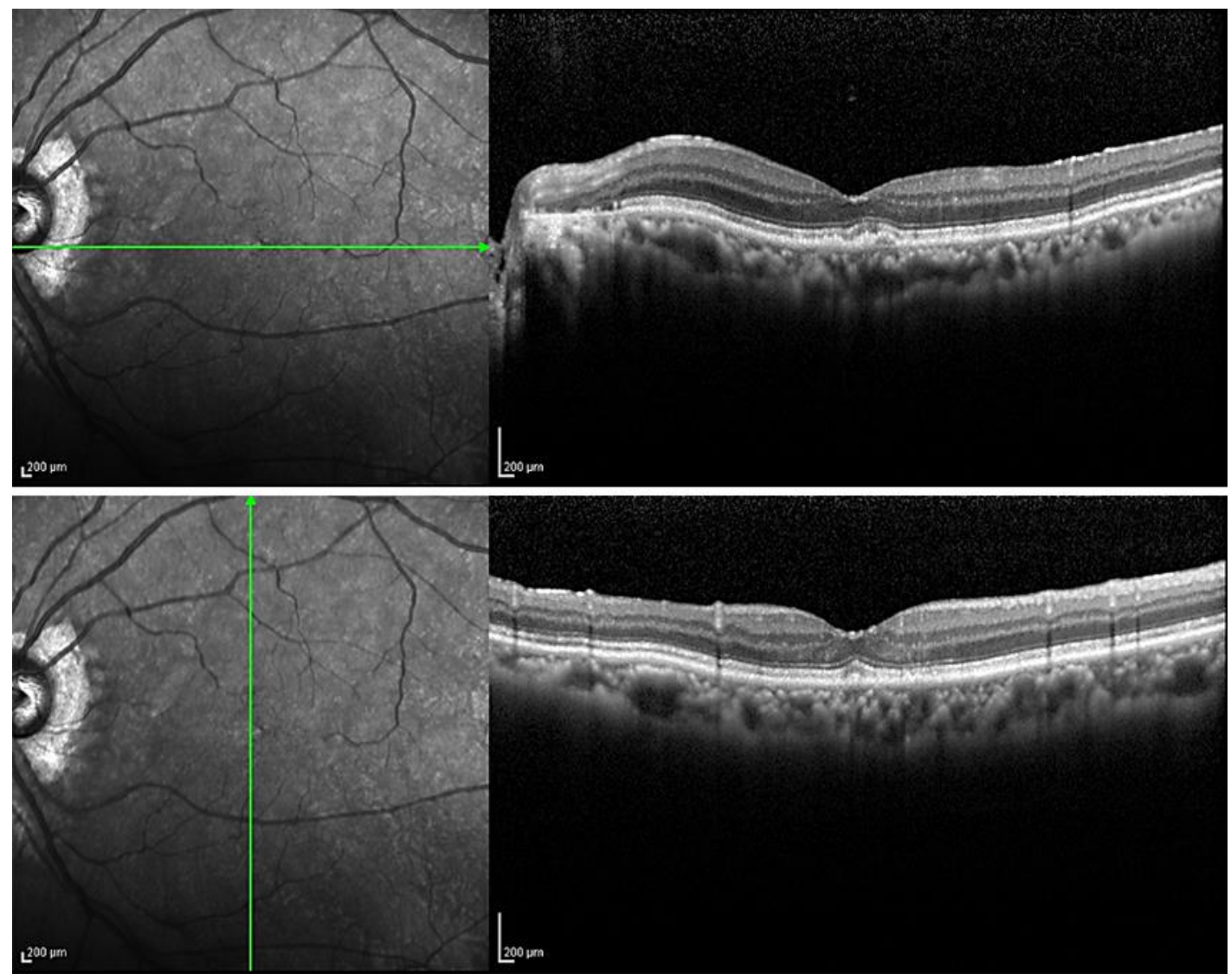

Fig. 3. OCT scans of the recovered eye. IRF completely disappeared and macular anatomy had recovered 31 months after surgery. Visual acuity had also improved to 20/20 Snellen acuity. OCT, optical coherence tomography; IRF, intraretinal fluid. 